九州大学学術情報リポジトリ

Kyushu University Institutional Repository

\title{
Different roles of s100p overexpression in intrahepatic cholangiocarcinoma :
}

Carcinogenesis of perihilar type and aggressive behavior of peripheral type

Aishima, Shinichi

Department of Anatomic Pathology, Graduate School of Medical Sciences, Kyushu University

Fujita, Nobuhiro

Department of Anatomic Pathology, Graduate School of Medical Sciences, Kyushu University

Mano, Yohe i

Department of Anatomic Pathology, Graduate School of Medical Sciences, Kyushu University

Kubo, Yuichiro

Department of Anatomic Pathology, Graduate School of Medical Sciences, Kyushu University

他

http://hdl. handle. net/2324/25471

出版情報: American Journal of Surgical Pathology. 35 (4)，pp.590-598，2011-04. Lippincott Williams \& Wilkins

バージョン :

権利関係: (C) 2011 by Lippincott Williams \& Wilkins 
Different roles of S100P overexpression in intrahepatic cholangiocarcinoma; Carcinogenesis of perihilar type and aggressive behavior of peripheral type

Running title: S100P expression in intrahepatic cholangiocarcinoma

Shinichi Aishima, ${ }^{1)}$ Nobuhiro Fujita, ${ }^{1)}$ Yohei Mano, ${ }^{1)}$ Yuichiro Kubo, ${ }^{1)}$ Yuki Tanaka, ${ }^{1)}$ Akinobu Taketomi, ${ }^{2)}$ Ken Shirabe, ${ }^{2)}$ Yoshihiko Maehara, ${ }^{2)}$ Yoshinao Oda ${ }^{1)}$

${ }^{1)}$ Department of Anatomic Pathology, Graduate School of Medical Sciences, Kyushu University, Fukuoka 812-8582, Japan

${ }^{2)}$ Department of Surgery and Science, Graduate School of Medical Sciences, Kyushu University, Fukuoka 812-8582, Japan

Correspondence author: Shinichi Aishima, M.D., Anatomic Pathology, Kyushu

University, 3-1-1, Maidashi, Higashi-ku, Fukuoka 812-8582, Japan

E-mail: saish@surgpath.med.kyushu-u.ac.jp

Tel.: +81-92-642-6061; Fax: +81-92-642-5968 


\section{Abstract}

S100P is expressed in several kinds of malignant tumors. Intracellular S100P interacts with ezrin, and extracellular S100P activates the receptor for advanced glycation end-products (RAGE). However, little is known about the biological significance of $\mathrm{S} 100 \mathrm{P}$ and related protein in cholangiocarcinoma. Biliary intraepithelial neoplasia (BillN) is a precursor lesion of hilar or perihilar cholangiocarcinoma. We examined S100P, ezrin, and RAGE expression in 39 biliary intraepithelial neoplasia (BilIN) and 110 intrahepatic cholangiocarcinoma (ICC) cases, and analyzed its relation with clinicopathological factors and outcomes. S100P expression increased from reactive epithelium to low-grade BilIN to high-grade BilIN. S100P and ezrin expression rates in perihilar type ICC were higher than those in peripheral type ICC $(p<0.0001, p=0.0008$, respectively). S100P nuclear expression in peripheral type ICC was significantly correlated with vascular invasion $(\mathrm{p}=0.0209)$, lymphatic invasion $(\mathrm{p}=0.0003)$, and lymph node metastasis $(\mathrm{p}=0.003)$. S100P and ezrin expression was significantly correlated. S100P-positive and ezrin-positive cases indicate shorter survival in survival analysis of peripheral type $(\mathrm{p}=0.001, \mathrm{p}=0.0728$, respectively). Our results suggest that S100P-ezrin signaling have different roles of carcinogenesis of perihilar ICC and aggressive course of peripheral ICC. 


\section{Introduction}

The S100 proteins contain 2 EF-hand calcium-binding motifs that mediate $\mathrm{Ca}^{2+}$-dependent signal transduction. $\mathrm{S} 100$ proteins are localized in the nucleus and/or cytoplasm of a wide range of cells, and are involved in the regulation of cell-cycle progression and differentiation. ${ }^{14} \mathrm{~S} 100 \mathrm{P}$ protein is small isoform of the $\mathrm{S} 100$ protein family and is isolated from human placenta. ${ }^{10}$ Overexpression of $\mathrm{S} 100 \mathrm{P}$ has been detected in several tumors, including pancreatic, ${ }^{6,13,26,29,37}$ colon, ${ }^{17}$ breast, ${ }^{18}$ prostate, ${ }^{9}$ lung, ${ }^{32}$ and ovarian cancers. ${ }^{34}$

S100P functions as both extracellular and intracellular signaling molecules. Intracellular S100P can interact with the cytoskeletal protein ezrin by $\mathrm{Ca}^{2+}$-dependent regulation. ${ }^{24}$ Ezrin is a member of the ERM (ezrin/radixin/moesin) family, linking the plasma membrane and the actin cytoskeleton; ${ }^{36}$ it also plays a role in the regulation of cell migration and cell proliferation. ${ }^{8,39}$ In several human malignant tumors, ezrin has been implicated in tumor cell metastasis and malignant progression. ${ }^{4,5,15,16,23,42}$ Extracellular S100P can activate receptors for advanced glycation end-products (RAGE), which is known as a multiligand transmembrane receptor of the immunoglobulin superfamily. ${ }^{5,20,27}$ RAGE is widely expressed in normal tissue, ${ }^{12}$ and has been 
considered to affect the characteristic differences in various cancer cells. ${ }^{21,25,27,35}$ Recent studies have shown that S100P-RAGE signaling plays a major role in tumor development. ${ }^{5,6,17}$

Intrahepatic cholangiocarcinoma (ICC) shows the second most common primary liver cancer. Despite recent advances, the molecular mechanisms involved in carcinogenesis and the development of ICC remain poorly understood. Biliary intraepithelial neoplasia (BillN) is considered to be a precursor or non-invasive lesion of invasive cholangiocarcinoma of the hilar or perihilar region. ${ }^{41}$ Lin $\mathrm{F}$ and colleagues have observed that S100P is overexpressed in $90 \%$ of pancreatic intraepithelial neoplasia (PanIN) cases, $100 \%$ of pancreatic adenocarcinoma cases, and $61 \%$ of cholangiocarcinoma cases. ${ }^{26}$

In the present study, we assessed the expression of S100P, ezrin, and RAGE in BillN and invasive ICC, and investigated its role in cholangiocarcinogenesis and tumor progression.

\section{Materials and Methods}

\section{Tissue samples}

The tumors and biliary lesions of the study were surgically resected and diagnosed at 
the Department of Anatomic Pathology of Kyushu University from 1985 to 2008. Our study protocol conformed to the ethical guidelines of the 1975 Declaration of Helsinki. For strict privacy protection, identifying information for all samples was removed before analysis. We reviewed sections from 45 cases of hepatolithiasis and 5 cases of primary sclerosing cholangitis, and selected the biliary reactive epithelium $(n=11$, hepatolithiasis) and biliary intraepithelial neoplasia (BilIN) (n=39, 34 hepatolithiasis and 5 primary sclerosing cholangitis). The histopathological definition of BillN was based on the classification proposed by Zen Y. et al. ${ }^{40}$ Reactive epithelium was defined as the mildly hyperplastic epithelium with intraepithelial neutrophils and lymphocytic infiltration, lacking a definite disturbance of cell polarity (Fig. 1A). BillN-1 showed mild cellular atypia with enlarged nuclei and a minimal disturbance of cell polarity (n=20, Fig. 1B). BillN-2 showed evident cellular atypia with a focal disturbance of cell polarity (n=12, Fig. 1E). BillN-3 showed definite cellular atypia, or nuclear pleomorphism, or completely loss of cell polarity (n=7, Fig. F). BillN lesion and reactive epithelium were graded by two pathologists (S.A. and Y. M.), respectively. Then, we discussed the grade of the lesion. The definition of intrahepatic cholangiocarcinomas (ICCs) was based on the classification proposed by the World Health Organization, ${ }^{38}$ and 110 cases of invasive ICC were reviewed. The ICCs were 
divided into the perihilar type ( $\mathrm{n}=41$, Fig. 2A), which is involved in a large bile duct, or the peripheral type $(n=69$, Fig. 2B), which is involved in smaller than segmental branches based on gross and histologic classifications developed from previous study. ${ }^{3}$ The median age of the patients with ICC was 64 years (range, 33 to 90 years); the group included 67 men and 43 women. The median tumor size was $4 \mathrm{~cm}$ (range, 1 to $12 \mathrm{~cm}$ ). Vascular invasion, lymphatic invasion, and lymph node metastasis were observed in 75 cases $(68 \%), 56$ cases $(51 \%)$, and 28 cases (25\%) of ICC, respectively.

\section{Immunohistochemical staining and evaluation}

Tumor samples were fixed with $10 \%$ formaldehyde, embedded in paraffin, and sectioned into 4-um-thick slices. Immunohistochemical staining was performed by the streptavidin-biotin-peroxidase method (Histofine, Nichirei, Tokyo). Primary antibodies used in this study were goat monoclonal anti-S100P (1:200 dilution; Santa Cruz Biotechnology), rabbit polyclonal anti-Ezrin (1:200 dilution; Cell signaling technology), and goat monoclonal anti-RAGE (1:500 dilution; Santa Cruz Biotechnology). After the inhibition of endogenous peroxidase in a $3 \% \mathrm{H}_{2} \mathrm{O}_{2}$-methanol solution for $20 \mathrm{~min}$ and antigen retrieval (microwave irradiation in citrate buffer (pH6.0) for both antibodies), sections were exposed to each primary antibody at $4{ }^{\circ} \mathrm{C}$ overnight. The sections were 
then reacted in 3,3'-diaminobenzidine, counterstained with hematoxylin, and mounted. Evaluation of the immunohistochemical results was scored by two pathologists (Y.M. and S.A.) without knowledge of the clinical data.

Immunohistochemical staining was evaluated in the area showing the highest degree of dysplasia in each BilIN. To evaluate the expression of ICC, representative fields, including cancer and non-cancerous areas, were selected. The proportion of positive cells was semiquantitatively measured using the following scale according to the percentage of positive tumor cells: 0 ( $<1 \%$ positive cells $), 1+(1-10 \%$ positive cells $), 2+$ (11-50\% positive cells), $3+(>51 \%$ positive cells). The expression of S100P in dysplastic or cancer cells was defined as positive when the nucleus or cytoplasm was stained to show more than $11 \%$ dysplastic or cancer cells, and separately evaluated both nuclear and cytoplasmic staining. For the survival analysis of ICC cases, S100P total expression was classified into negative group $(0 / 1+$ nuclear expression and $0 / 1+$ cytoplasmic expression) and positive group $(2+/ 3+$ nuclear expression and/or $2+/ 3+$ cytoplasmic expression). The expression of RAGE and ezrin in dysplastic cells or cancer cells was defined as positive when the cytoplasm was stained to show more than $11 \%$ tumor cells.

\section{Statistical analysis}


Statistical analysis of group differences was performed by the chi-squared test, Fisher's exact test, and Mann-Whitney U-test. The Kaplan-Meier method was used for survival analysis, and a comparison was made based on the log-rank test. $P<0.05$ was considered statistically significant.

\section{Results}

Immunohistochemical expression in reactive epithelium, BilIN, and invasive ICC

Immunohistochemical scores and positive rates are summarized in Table 1. S100P immunoreactivity was absent in nucleus, but focally positive in cytoplasm of reactive epithelium, while S100P was extensively observed in infiltrating neutrophils (Fig 1C). In 20 cases of BillN-1, S100P was observed in the nucleus $(3 / 20 ; 15 \%)$ and in cytoplasm (9/20; 45\%) (Fig. 1D). In 12 cases of BilIN-2, 9 (75\%) of nuclear stain and $8(67 \%)$ of cytoplasmic stain were observed (Fig. G), and all 7 cases of BillN-3 showed diffuse nuclear and cytoplasmic stains (Fig. H). Nuclear and cytoplasmic S100P were observed in 28/41 (68\%) and 23/41 (63\%) of perihilar type ICC (Fig. 2C), while they were observed in 8/69 (12\%) and in 9/69 (13\%) of peripheral type ICC (Fig. 2D). Nuclear S100P expression was increased from reactive epithelium to BilIN-2 (Fig. 3A). 
Cytoplasmic S100P expression was gradually increased reactive epithelium to BilIN-3 and decreased from BilIN-3 to perihilar type (Fig. 3B). Nuclear and cytoplasmic S100P expression rate was significantly higher in perihilar type than in peripheral type ICC $(\mathrm{p}<0.0001)($ Fig. 3A, B).

Positive staining for ezrin was observed in 4/11 (36\%) cases of reactive epithelium, 5/20 (25\%) of BillN-1, 9/12 (75\%) of BillN-2, 6/7 (86\%) of BillN-3, 20/41 (49\%) perihilar type ICC (Fig.2E), and 14/69 (20\%) of peripheral type ICC (Fig. 2F). Ezrin expression of BilIN-2 was higher than that of BillN-1 ( $\mathrm{p}=0.0004)$. Ezrin expression of perihilar type was higher than that of peripheral type ( $\mathrm{p}=0.0008$, Fig. $3 \mathrm{C})$.

RAGE immunoreactivity was weakly observed in some peribiliary glands or liver parenchyma. RAGE expression was observed in 1/11 (9\%) of reactive epithelium, 2/20 (10\%) of BillN-1, 3/12 (25\%) of BillN-2, none (0\%) of BilIN-3, 7/41 (17\%) of perihilar type ICC (Fig. 2G), and 17/69 (25\%) of peripheral type ICC (Fig. 2H, 3D).

\section{Immunohistochemical results and clinicopathological correlations in ICC}

In perihilar type ICC, no significant correlations were observed between clinicopathological parameters and immunohistochemical expression (Table 2). In peripheral type ICC, S100P nuclear expression was significantly higher in cases with 
vascular invasion $(\mathrm{p}=0.0209)$, lymphatic invasion $(\mathrm{p}=0.0003)$, and lymph node metastasis $(p=0.003$, Table 3$)$. S100P cytoplasmic examination also shows positive statistical correlations with lymphatic invasion $(\mathrm{p}=0.0231)$ and lymph node metastasis $(\mathrm{p}=0.006$, Table 3$)$.

The nuclear and cytoplasmic expression of S100P in ICC correlated with ezrin expression ( $\mathrm{p}=0.004, \mathrm{p}=0.0248$, respectively), while, there was no statistical relationship between S100P expression and RAGE expression (Table 4).

\section{Protein expression and patient outcomes}

Patient survival was evaluated by follow-up for a mean period of 3.13 years. The survival rates for patients with ICC at 1,3 , and 5 years were $73.6 \%, 48.4 \%$, and $34.2 \%$, respectively. In patients with periphilar ICC, there was no statistical difference between the survival rate and three protein expression (Fig.4A, C, E). The cancer-specific survival rates of peripheral type ICC with positive total S100P expression were significantly lower than those of peripheral type ICC with negative total S100P expression ( $\mathrm{p}=0.001$, Fig. 4B). Peripheral type ICC with positive ezrin expression showed lower survival tendency than those with negative ezrin expression $(\mathrm{p}=0.0728$, Fig. 4D), but there was no statistical difference between the survival rate and RAGE 
expression ( $\mathrm{p}=0.9952$, Fig. 4F). Perihilar type ICC (Fig. 5A,C) and peripheral type ICC (Fig. 5B,D) shows significant survival differences with respect to the lymphatic invasion (Fig. 5A, p=0.0002, Fig. 5B, p<0.0001) and nodal metastasis (Fig. 5C, $\mathrm{p}=0.0004$, Fig. 5D, p<0.0001).

\section{Discussion}

The only curative treatment for cholangiocarcinoma is complete surgical resection, and no beneficial roles were demonstrated in chemo-radiotherapy. The high expression of S100P has been regarded as a potential target molecule for therapeutic applications; ${ }^{31}$ however, other studies have shown that S100P plays a critical role in resistance to chemotherapeutic agents. ${ }^{5,20}$ In this study, S100P was overexpressed in the aggressive peripheral type ICC with lymphatic invasion and lymph node metastasis in surgical samples. Peripheral ICC with positive S100P showed poorer prognosis after surgery than those with negative S100P. Our results suggest that S100P plays a role in cell invasion and metastasis in peripheral ICC, as suggested in the studies involving pancreatic carcinoma cells. ${ }^{6,37}$

Biliary intraepithelial neoplasia (BillN) is premalignant or non-invasive lesions of perihilar type ICC. ${ }^{3,41} \mathrm{~S} 100 \mathrm{P}$ and ezrin showed an increased protein expression from 
low-grade BilIN to high-grade BilIN. Our results suggest a critical role for S100P and ezrin in cholangiocarcinogenesis of the large bile duct. In contrast, peripheral type ICC, which is located in the periphery of the liver and is suggestive of a lesion arising from the small biliary epithelium, showed low positivity for S100P and ezrin expression. The molecular mechanism of S100P overexpression in ICC remained unclear in our study. IL-6 is increased in the serum of patients with cholangiocarcinoma ${ }^{28}$ and stimulates the growth of cholangiocarcinoma cells. ${ }^{30}$ In addition, IL-6 is a potent inducer of S100P in prostate cancer. ${ }^{19}$ Prostaglandin E2, which is expressed in hepatolithiasis, ${ }^{33}$ can induce S100P. ${ }^{11}$ Therefore, S100P may be upregulated by IL-6 or prostaglandin stimulation of inflammatory condition in carcinogenesis of periphilar cholangiocarcinoma.

The differences in positivity for S100P and ezrin between perihilar type ICC and peripheral type ICC support the concept of the different process of carcinogenesis in the two type of ICC. ${ }^{3}$ We have previously shown the significant biological differences between perihilar type ICC and peripheral type ICC in the expression of MUC5AC (mucus core protein), aquaporin-1 (water channel molecule), and intratumoral arterial vessel density. ${ }^{1,2}$ Together with the results indicating that more than $90 \%$ of pancreatic intraepithelial neoplasia (PanIN) and pancreatic invasive adenocarcinoma show positive S100P expression, ${ }^{26}$ perihilar type ICC may have same carcinogenetic pathway in 
pancreatic adenocarcinoma. $\underline{\text { S100P overexpression was related with carcinogenesis or }}$ early development of pancreatic cancer, ${ }^{26,29}$ whereas S100P expression of prostatic cancer $^{9}$ and ovarian cancer $^{34}$ was associated with aggressive tumor behavior and poor prognosis. In addition, ezrin expression of hepatocellular carcinoma (HCC) was associated with advanced stage and poor prognosis. ${ }^{23}$ Peripheral type ICC showed higher background of chronic hepatitis and cirrhosis, and similar behavior with HCC. ${ }^{3}$ These results suggest that the role of S100P and ezrin was different in the kinds of malignant tumor and two type of ICC.

Although extracellular S100P stimulates cell proliferation via the RAGE, ${ }^{5}$ Austermann J and colleagues have shown S100P-mediated activation of ezrin and suggested that the interaction between S100P and ezrin promotes a migration of tumor cells and metastatic potential. ${ }^{7,8}$ Our study has demonstrated that $\mathrm{S} 100 \mathrm{P}$ expression is significantly correlated with ezrin expression, not RAGE expression, in ICC tissue. RAGE expression tended to be higher in peripheral type ICC than perihilar type ICC. Because S100P protein was localized in the cytoplasm and/or nucleus of cancer cells, the hypothesis that extracellular S100P induces RAGE activation, was not confirmed in the resected specimen of ICC.

In conclusion, S100P and ezrin were found to be expressed in gradual increase from 
reactive epithelium to premalignant biliary neoplasia and invasive cholangiocarcinoma of the perihilar type, and they appear to contribute to tumor invasiveness and metastasis in peripheral ICC. Our results suggest that S100P-ezrin signaling have different roles of carcinogenesis of perihilar ICC and aggressive tumor behavior of peripheral ICC.

\section{References}

1. Aishima S, Iguchi T, Nishihara Y, et al. Decreased intratumoral arteries reflect portal tract destruction and aggressive characteristics in intrahepatic cholangiocarcinoma. Histopathology 2009;54:452-461.

2. Aishima S, Kuroda Y, Nishihara Y, et al. Down-regulation of aquaporin-1 in intrahepatic cholangiocarcinoma is related to tumor progression and mucin expression. Hum Pathol 2007;38:1819-1825.

3. Aishima S, Kuroda Y, Nishihara Y, et al. Proposal of Progression Model for Intrahepatic Cholangiocarcinoma: Clinicopathologic Differences Between Hilar Type and Peripheral Type. Am J Surg Pathol 2007;31:1059-1067.

4. Akisawa N, Nishimori I, Iwamura T, et al. High levels of ezrin expressed by human pancreatic adenocarcinoma cell lines with high metastatic potential. Biochem Biophys Res Commun 1999;258:395-400. 
5. Arumugam T, Simeone DM, Schmidt AM, et al. S100P stimulates cell proliferation and survival via receptor for activated glycation end products (RAGE). J Biol Chem 2004;279:5059-5065.

6. Arumugam T, Simeone DM, Van Golen K, et al. S100P promotes pancreatic cancer growth, survival, and invasion. Clin Cancer Res 2005;11:5356-5364.

7. Austermann J, Nazmi AR, Heil A, et al. Generation and characterization of a novel, permanently active S100P mutant. Biochim Biophys Acta 2009;1793:1078-85.

8. Austermann J, Nazmi AR, Müller-Tidow C, et al. Characterization of the Ca2+ -regulated ezrin-S100P interaction and its role in tumor cell migration. J Biol Chem 2008;283:29331-29340.

9. Basu GD, Azorsa DO, Kiefer JA, et al. Functional evidence implicating S100P in prostate cancer progression. Int J Cancer 2008;123:330-339.

10. Becker T, Gerke V, Kube E, et al. S100P, a novel Ca(2+)-binding protein from human placenta. cDNA cloning, recombinant protein expression and $\mathrm{Ca} 2+$ binding properties. Eur J Biochem 1992; 207:541-547.

11. Chandramouli A, Mercado-Pimentel ME, Hutchinson A, et al. The induction of S100p expression by the Prostaglandin E(2) (PGE(2))/EP4 receptor signaling pathway in colon cancer cells. Cancer Biol Ther 2010;10;1056-1066. 
12. Cheng C, Tsuneyama K, Kominami R, et al. Expression profiling of endogenous secretory receptor for advanced glycation end products in human organs. Mod Pathol 2005;18:1385-1396.

13. Crnogorac-Jurcevic T, Missiaglia E, Blaveri E, et al. Molecular alterations in pancreatic carcinoma: expression profiling shows that dysregulated expression of S100 genes is highly prevalent. J Pathol 2003;201:63-74.

14. Donato R. S100: a multigenic family of calcium-modulated proteins of the EF-hand type with intracellular and extracellular functional roles. Int J Biochem Cell Biol 2001; 33:637-668.

15. Endo K, Kondo S, Shackleford J, et al. Phosphorylated ezrin is associated with EBV latent membrane protein 1 in nasopharyngeal carcinoma and induces cell migration.Oncogene 2009;28:1725-1735.

16. Elzagheid A, Korkeila E, Bendardaf R, et al. Intense cytoplasmic ezrin immunoreactivity predicts poor survival in colorectal cancer.Hum Pathol 2008;39:1737-1743.

17. Fuentes MK, Nigavekar SS, Arumugam T, et al. RAGE activation by S100P in colon cancer stimulates growth, migration, and cell signaling pathways. Dis Colon Rectum 2007;50:1230-1240. 
18. Guerreiro Da Silva ID, Hu YF, Russo IH, et al. S100P calcium-binding protein overexpression is associated with immortalization of human breast epithelial cells in vitro and early stages of breast cancer development in vivo. Int J Oncol 2000;16:231-240.

19. Hammacher A, Thompson EW, Williams ED: Interleukin-6 is a potent inducer of S100P, which is up-regulated in androgenrefractory and metastatic prostate cancer. Int J Biochem Cell Biol 2005;37:442-450.

20. Heizmann CW, Ackermann GE, Galichet A. Pathologies involving the S100 proteins and RAGE. Subcell Biochem 2007;45:93-138.

21. Hiwatashi K, Ueno S, Abeyama K, et al. A novel function of the receptor for advanced glycation end-products (RAGE) in association with tumorigenesis and tumor differentiation of HCC. Ann Surg Oncol 2008;15:923-933.

22. Jiang F, Shults K, Flye L, et al. S100P is selectively upregulated in tumor cell lines challenged with DNA cross-linking agents. Leuk Res 2005;29:1181-1190.

23. Kang YK, Hong SW, Lee H, Kim WH. Prognostic implications of ezrin expression in human hepatocellular carcinoma. Mol Carcinog. 2010;49:798-804.

24. Koltzscher M, Neumann C, König S, et al.Ca2+-dependent binding and activation of dormant ezrin by dimeric S100P. Mol Biol Cell 2003;14:2372-2384. 
25. Kuniyasu H, Oue N, Wakikawa A, et al. Expression of receptors for advanced glycation end-products (RAGE) is closely associated with the invasive and metastatic activity of gastric cancer. J Pathol 2002;196:163-170.

26. Lin F, Shi J, Liu H, et al. Diagnostic utility of S100P and von Hippel-Lindau gene product (pVHL) in pancreatic adenocarcinoma-with implication of their roles in early tumorigenesis. Am J Surg Pathol 2008;32:78-91.

27. Logsdon CD, Fuentes MK, Huang EH, et al. RAGE and RAGE ligands in cancer. Curr Mol Med 2007;7:777-789.

28. Mott JL, Gores GJ. Targeting IL-6 in cholangiocarcinoma therapy. Am J Gastroenterol 2007;102:2171-2172.

29. Ohuchida K, Mizumoto K, Egami T, et al. S100P is an early developmental marker of pancreatic carcinogenesis. Clin Cancer Res 2006;12:5411-5416.

30. Park J, Tadlock L, Gores GJ, et al. Inhibition of interleukin 6-mediated mitogen-activated protein kinase activation attenuates growth of a cholangiocarcinoma cell line. Hepatology 1999;30:1128-1133.

31. Parkkila S, Pan PW, Ward A, et al. The calcium-binding protein S100P in normal and malignant human tissues. BMC Clin Pathol 2008;8:2. 
32. Rehbein G, Simm A, Hofmann HS, et al. Molecular regulation of S100P in human lung adenocarcinomas. Int J Mol Med 2008;22:69-77.

33. Shoda J, Ueda T, Kawamoto T, et al. Prostaglandin E receptors in bile ducts of hepatolithiasis patients and the pathobiological significance for cholangitis. Clin Gastroenterol Hepatol 2003;1:285-296.

34. Surowiak P, Maciejczyk A, Materna V, et al. Unfavourable prognostic significance of S100P expression in ovarian cancers. Histopathology 2007;51:125-128.

35. Tateno T, Ueno S, Hiwatashi K, et al. Expression of receptor for advanced glycation end products (RAGE) is related to prognosis in patients with esophageal squamous cell carcinoma. Ann Surg Oncol 2009;16:440-446.

36. Vaheri A, Carpén O, Heiska L, et al. The ezrin protein family: membrane-cytoskeleton interactions and disease associations. Curr Opin Cell Biol 1997;9:659-666.

37. Whiteman HJ, Weeks ME, Dowen SE, et al. The role of S100P in the invasion of pancreatic cancer cells is mediated through cytoskeletal changes and regulation of cathepsin D. Cancer Res 2007;67:8633-8642.

38. Wittekind C, Fischer HP, Ponchon T. Intrahepatic cholangiocarcinoma. In: Nakanuma Y, Sripa B, Vatanasapt V, et al, eds. World Health Organization 
Classification of tumours: Pathology and Genetics of Tumours of the Digestive System. Lyon, France: IARC Press, 2000:173-180.

39. Xie JJ, Xu LY, Xie YM, et al. Roles of ezrin in the growth and invasiveness of esophageal squamous carcinoma cells. Int J Cancer 2009;124:2549-2558.

40. Zen Y, Adsay NV, Bardadin K, et al. Biliary intraepithelial neoplasia: an international interobserver agreement study and proposal for diagnostic criteria. Mod Pathol 2007;20:701-709.

41. Zen Y, Sasaki M, Fujii T, et al. Different expression patterns of mucin core proteins and cytokeratins during intrahepatic cholangiocarcinogenesis from biliary intraepithelial neoplasia and intraductal papillary neoplasm of the bile duct--an immunohistochemical study of 110 cases of hepatolithiasis. J Hepatol 2006;44:350-358.

42. Zhang Y, Hu MY, Wu WZ, et al. The membrane-cytoskeleton organizer ezrin is necessary for hepatocellular carcinoma cell growth and invasiveness. J Cancer Res Clin Oncol 2006;132:685-697.

\section{Figure legends}




\section{Figure 1}

Representative cases of reactive epithelium (A), BillN-1 (B), BillN-2 (E), and BillN-3 (F) by HE stain. S100P expression was observed in infiltrating neutrophils, but not in the reactive epithelium (C). S100P expression was observed only in cytoplasm of BillN-1 (D), and BilIN-2 showed nuclear and cytoplasmic expression (G). Strong nuclear and cytoplasmic expression of S100P was observed diffusely in BillN-3 (H).

\section{Figure 2}

Perihilar type ICC showed invasive tubular adenocarcinoma (A), and peripheral type ICC showed moderately differentiated adenocarcinoma with irregular small tubules (B). Large tubular carcinoma of perihilar type was positive for S100P (nuclear and cytoplasmic) (C) and ezrin (E), but negative for RAGE (G). Small tubular carcinoma of peripheral type was negative for S100P (D) and ezrin (F), but positive for RAGE (H).

\section{Figure 3}

Results of nuclear scores (A) and cytoplasmic scores (B) of S100P, scores of ezrin (C), and scores of RAGE (D). Nuclear S100P expression was increased from reactive to BilIN-2. Cytoplasmic S100P expression was gradually increased reactive to BilIN-3 and decreased from BilIN-3 to perihilar type. Ezrin expression of BillN-2 was higher than that of BilIN1. S100P and ezrin expression of perihilar type were higher than those of 
peripheral type.

\section{Figure 4}

Survival curves according to the immunohistochemical results were created based on immunohistochemistry in cases of perihilar type (A,C,E) and peripheral type (B,D,F). (B) $P=0.001, \mathrm{~S} 100 \mathrm{P}$-positive patients versus $\mathrm{S} 100 \mathrm{P}-$ negative patients. (D) $P=0.0728$, Ezrin-positive patients versus ezrin-negative patients.

\section{Figure 5}

Both perihilar type $(A, C)$ and peripheral type $(B, D)$ shows significant survival differences with respect to the lymphatic invasion $(A, B)$ and nodal metastasis $(C, D)$. 